\title{
Postcolumn Fluorometric Determination of Water-Soluble Organic Phosphates by High-Performance Liquid Chromatography
}

\author{
Yoshihiko IKEGUCHI and Hiroshi NAKAMURA ${ }^{\dagger}$ \\ Department of Analytical Chemistry, Faculty of Pharmaceutical Sciences, Science University of Tokyo, \\ Ichigaya-funagawara-machi, Shinjuku, Tokyo 162-0826, Japan
}

Keywords Reversed-phase HPLC, anion-exchange HPLC, postcolumn fluorescence detection, on-line hydrolysis, phospho-amino acids, phosphorylated peptides, phosphorylated carbohydrates

A variety of water-soluble organic phosphates are playing important roles in vivo, e.g. phosphorylated proteins in signal transduction and phosphorylated sugars in the glycolytic pathway. In contrast, hydrophobic organic phosphates, represented by phospholipids, are generated from or hydrolyzed to hydrophilic organic phosphates and they are also implicated in various cellular responses. Therefore, an analytical method enabling determination of both hydrophobic and hydrophilic organic phosphates is highly desired as a useful tool in the biomedical field. Only mass spectrometry (MS) can provide the phosphate-selective HPLC determination for both types of phosphates, such as phospholipids ${ }^{1}$, phosphorylated carbohydrates ${ }^{2}$ and phosphorylated protein/peptides. ${ }^{3}$

We previously reported on the development of an HPLC system with a postcolumn fluorescence detector for the determination of phospholipids as a model compound of hydrophobic organic phosphates, based on a series of hydrolyses under UV-irradiation in the presence of $\mathrm{TiO}_{2}$, phosphomolybdic acid and thiaminethiocrome reactions. ${ }^{4}$ In this research, we succeeded in reversed-phase or anion-exchange HPLC separation of water-soluble organic phosphates based on the previous postcolumn fluorescence detection system ${ }^{4}$ in which only the composition of the hydrolysis reagent was changed.

\section{Experimental}

\section{Materials and reagents}

Reagents described below were all guaranteed grade and were used without further purification. Samples used were dissolved in water purified on a Milli ROMilli Q system (Millipore, Bedford, MA, USA).

\section{HPLC system with postcolumn fluorescence detection}

The HPLC system used in this experiment is basically

$\doteqdot$ To whom correspondence should be addressed. the same as in our previous method ${ }^{4}$ for the determination of phospholipids and is briefly outlined below. The water-soluble organic phosphates were separated on a CAPCELLPAK $\mathrm{C}_{18}$ column $(4.6 \mathrm{~mm}$ i.d. $\times 250 \mathrm{~mm}$, Shiseido, Tokyo, Japan) or an Asahipak NH2P-50 (4.6 $\mathrm{mm}$ i.d. $\times 250 \mathrm{~mm}$, Shodex, Tokyo, Japan). The samples separated on each column were introduced into the hydrolysis coil and irradiated with the UV-lamp in order to liberate orthophosphate. The resultant orthophosphate was reacted with a molybdenum reagent to form the phosphomolybdic acid, which was then reacted with the alkaline thiamine reagent. Finally, the fluorescence intensity of the thiochrome converted from thiamine by phosphomolybdic acid was measured at the excitation wavelength of $380 \mathrm{~nm}$ and emission wavelength of $442 \mathrm{~nm}$.

\section{Peptide phosphorylation}

A mixture of two peptides, kemptide (Leu-Arg-ArgAla-Ser-Leu-Gly; Sigma, St. Louis, MO, USA) and malantide (Arg-Thr-Lys-Arg-Ser-Gly-Ser-Val-Tyr-GluPro-Leu-Lys-Ile; Sigma), was dissolved at a concentration of $200 \mu \mathrm{M}$ each in $20 \mathrm{mM}\left(\mathrm{CH}_{3} \mathrm{COOH}\right)_{2} \mathrm{Mg}, 10$ $\mu \mathrm{M}$ cAMP, $25 \mathrm{mM}$ 2-morpholino-ethanesulfonic acid (MES) (pH 6.9), $1 \mathrm{mM}$ ATP and $85 \mu \mathrm{g} \mathrm{ml}^{-1}$ cAMPdependent protein kinase catalytic subunit (Sigma), and incubated at $37^{\circ} \mathrm{C}$ for $1 \mathrm{~h}$. An aliquot $(20 \mu \mathrm{l})$ of the reaction mixture was injected to the HPLC system. The underlined serine residues in kemptide and malantide are phosphorylated in the enzymatic reaction described above.

\section{Results and Discussion}

Hydrolysis conditions for FIA and reversed-phase HPLC separation of phospho-amino acids

A hydrolysis reagent for the determination of phospho-amino acids, i.e. phospho-serine (P-Ser), phosphothreonine (P-Thr) and phospho-tyrosine (P-Tyr), was optimized with flow injection analysis (FIA) using 
Table 1 Effect of additives on hydrolysis of phosphoamino acids

\begin{tabular}{|c|c|c|c|c|}
\hline \multicolumn{2}{|c|}{ Additives } & \multicolumn{3}{|c|}{ Hydrolysis, \% } \\
\hline $10 \%$ Acetonitrile & $0.2 \%$ Sodium deoxycholate & P-Tyr & P-Thr & P-Ser \\
\hline- & $+{ }^{a}$ & 35.9 & 37.1 & 39.1 \\
\hline+ & + & 66.6 & 61.2 & 69.6 \\
\hline+ & - & 76.8 & 75.5 & 78.4 \\
\hline
\end{tabular}

a. Hydrolysis reagent as a control contains $2 \mathrm{mg} \mathrm{l}^{-1} \mathrm{TiO}_{2}, 2 \% \mathrm{SDS}, 0.2 \%$ sodium deoxycholate and $0.1 \%$ TFA.

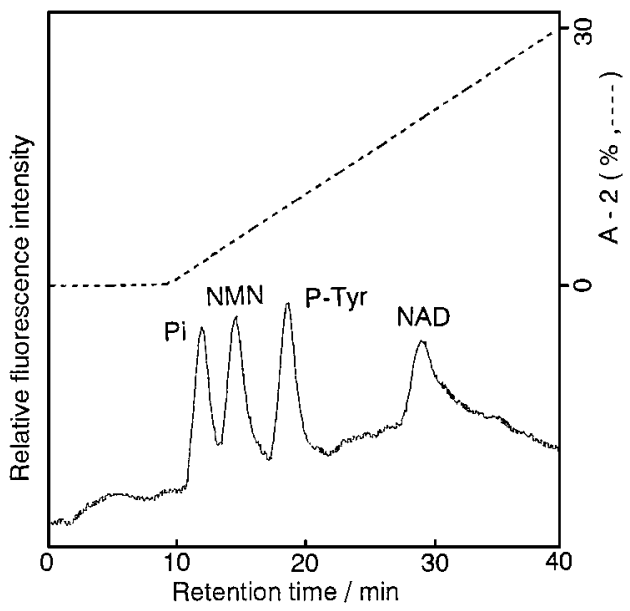

Fig. 1 Reversed-phase HPLC profile of some water-soluble organic phosphates. Column, Capcellpak $\mathrm{C}_{18}$; column temperature, $40^{\circ} \mathrm{C}$; eluent, A-1: acetonitorile/water/TFA (0/ 100/0.1, by volume); A-2: acetonitorile/water/TFA (70/30/ $0.1)$; elution program, (1) isocratic $(0-10 \mathrm{~min}), \mathrm{A}-1 / \mathrm{A}-2$ (100/0), (2) linear gradient $(10-40 \mathrm{~min}), \mathrm{A}-1 / \mathrm{A}-2(100 / 0)$ to A-1/A-2 (70/30); injected amount, 1 nmol each.

$0.1 \%$ trifluoroacetic acid (TFA) as a carrier solvent, where the column was deleted from our HPLC system ${ }^{4}$ with postcolumn fluorescence detection for phospholipids. The efficiency of hydrolysis reaction was calculated by taking a peak height of orthophosphate $(\mathrm{Pi})$ as 100. When the hydrolysis reagent for the phospholipid determination ${ }^{4}$ containing $2 \mathrm{mg} \mathrm{l}^{-1} \mathrm{TiO}_{2}, 2 \%(\mathrm{w} / \mathrm{v}) \mathrm{SDS}$, $0.2 \%(\mathrm{w} / \mathrm{v})$ sodium deoxycholate and $0.1 \%(\mathrm{v} / \mathrm{v})$ TFA was used, the hydrolysis rates of water-soluble organic phosphates were $35.1-39.1 \%(n=3)$. However, when $10 \%(\mathrm{v} / \mathrm{v}$, as a final concentration) acetonitrile was added and sodium deoxycholate was deleted from the hydrolysis reagent, the hydrolysis rates were found to increase up to $75.5-78.4 \%$ (Table 1). Consequently, 2 $\mathrm{mg}^{-1} \mathrm{TiO}_{2}, 2 \%$ SDS, $0.1 \%$ TFA and $10 \%$ acetonitrile was chosen as a composition for hydrolysis of phospho-amino acids. When $10 \mu \mathrm{l}$ was injected under the FIA condition, the calibration curves for the three phospho-amino acids were linear in the range $10-500 \mu \mathrm{M}$ (correlation coefficient, $0.993-0.997$ ) and the detection limits $(\mathrm{S} / \mathrm{N}=3)$ were $c a .50$ pmol. The relative standard deviation (RSD) of $1 \mathrm{nmol}$ each of the phospho-amino acid was between 2.76 and $3.59 \%(n=5)$.

Next, reversed-phase HPLC separation of some

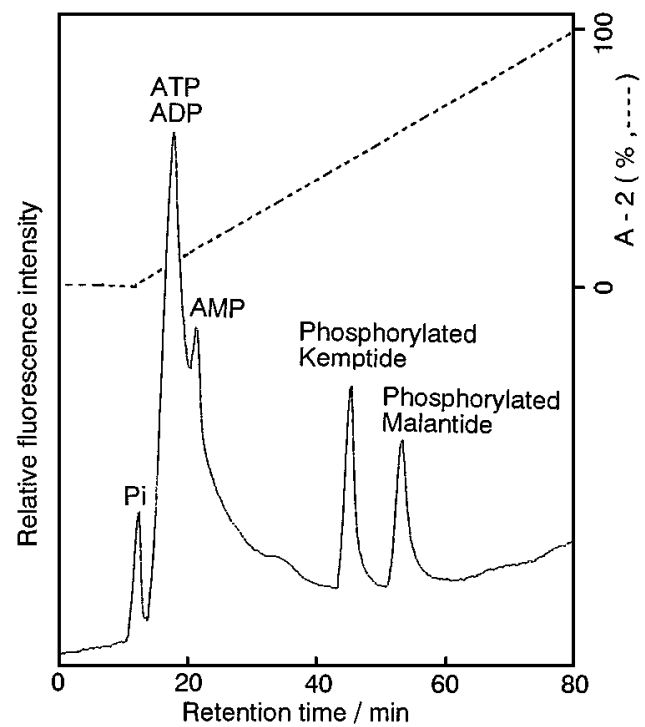

Fig. 2 Reversed-phase HPLC of phosphorylated peptides. The separation conditions were the same as in Fig. 2 except for linear gradient program; starting A-1/A-2 (100/0) at $t=10$ min and the ratio was changed to $(0 / 100)$ at $t=80 \mathrm{~min}$; injected volume, $20 \mu \mathrm{l}$.

water-soluble organic phosphates was attempted with a CAPCELLPAK $\mathrm{C}_{18}$ column using the above FIA condition. Figure 1 illustrates the reversed-phase separation of $1 \mathrm{nmol}$ each of $\mathrm{Pi}$, nicotinamide mononucleotide $(\mathrm{NMN})$, a metabolite of nicotinamide adenine dinucleotide (NAD), P-Tyr and NAD, whose cellular level decreases in the course of apoptosis ${ }^{5}$ prior to signal transduction. ${ }^{6}$ The calibration curves for P-Tyr, NMN and NAD were linear in the range $10-500 \mu \mathrm{M}$ (correlation coefficient, $0.993-0.996$ ) under the gradient elution program described in the legend of Fig. 1. The RSD of $1 \mathrm{nmol}$ each of the three compounds was between 2.43 and $3.98 \%(n=5)$. The detection limits $(S / N=3)$ obtained were $c a$. 50 pmol. This HPLC system was also applied to the analysis of phosphorylation products of two peptides (kemptide and malantide) (Fig. 2), indicating the applicability of the system to the determination of phosphorylated sites of phosphoproteins and to the measurement of kinase (phosphorylation enzyme) activity.

\section{Hydrolysis of phosphorylated carbohydrates}

The hydrolysis of phosphorylated carbohydrates was 
Table 2 Effect of additives on hydrolysis of phosphorylated carbohydrates

\begin{tabular}{|c|c|c|c|c|c|c|}
\hline \multicolumn{4}{|c|}{ Additives } & \multicolumn{3}{|c|}{ Hydrolysis, \% } \\
\hline $0.1 \%$ TFA & $50 \mathrm{mM}$ Boric acid & $0.5 \mathrm{mM}$ TBA & $10 \%$ Isopropanol & Glc-1-P & Rib-5-P & P-Ser \\
\hline+ & - & - & $-{ }^{a}$ & 20.0 & 22.8 & 75.5 \\
\hline- & + & + & - & 49.0 & 50.1 & 88.4 \\
\hline- & + & + & + & 85.9 & 82.0 & 79.5 \\
\hline
\end{tabular}

a. Hydrolysis reagent as a control contains $2 \mathrm{mg} \mathrm{l}^{-1} \mathrm{TiO}_{2}, 2 \%$ SDS, $0.1 \%$ TFA and $10 \%$ acetonitrile.

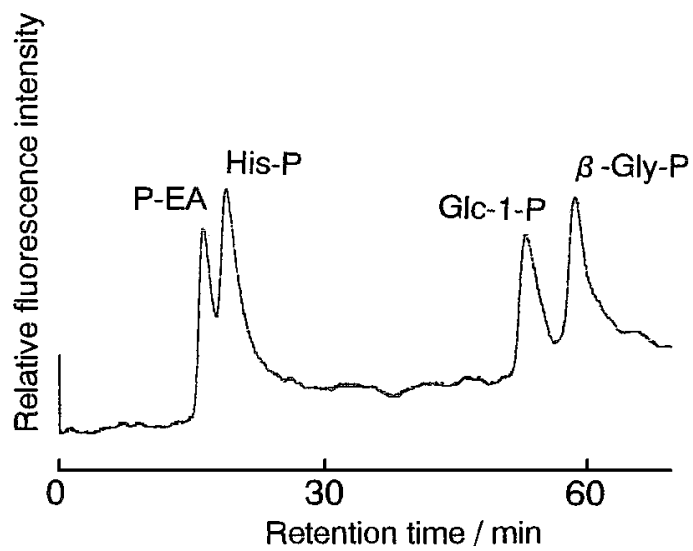

Fig. 3 Anion-exchange HPLC of water-soluble organic phosphates obtained with an isocratic elution. Column, Asahipak $\mathrm{NH} 2 \mathrm{P}-50$; column temperature, $50^{\circ} \mathrm{C}$; eluent, $160 \mathrm{mM} \mathrm{LiCl}$; injected amount, 2 nmol each.

also examined, as tested with the FIA system for phosphoamino acids. The hydrolysis reagent for phosphoamino acids did not show good efficiency in hydrolysis of glucose-1-phosphate (Glc-1-P) and ribose-5-phosphate (Rib-5-P) tested. As shown in Table 2, the hydrolysis rates of Glc-1-P and Rib-5-P were found to increase up to $85.9 \%$ and $82.0 \%$ respectively, when TFA was deleted and $50 \mathrm{mM}$ boric acid and $0.5 \mathrm{mM}$ tetrabutyl ammonium bromide (TBA) and $10 \%(\mathrm{v} / \mathrm{v})$ isopropanol were added to the established hydrolysis reagent containing $2 \mathrm{mg} \mathrm{l}^{-1} \mathrm{TiO}_{2}, 2 \% \mathrm{SDS}, 0.1 \%$ TFA and $10 \%$ acetonitrile. Boric acid as a cheleting reagent with carbohydrates, TBA as an ion-pair reagent for phosphorylated carbohydrates and isopropanol as another organic solvent had effect on hydrolysis for the tested compounds, as acetonitrile as an organic solvent did for phosphoamino acids (Table 1). Taken together, the results shown in Table 2 indicated that hydrolysis rates for water-soluble organic phosphates tested in SDS micelle surroundings, where SDS served to increase sensitivity in the system, were affected by SDS not only in photolysis catalytic activity of $\mathrm{TiO}_{2}$ under UV-irradiation, but also in some interactions of SDS micelle surroundings with organic phosphates them- selves or their complexes with borates. When $10 \mu \mathrm{l}$ was injected under this FIA condition, the calibration curves for Glc-1-P and Rib-5-P were linear in the range $20-500 \mu \mathrm{M}$ (correlation coefficient, 0.994 and 0.993, respectively), where the RSD in determination of 1 nmol each of organic phosphates was 3.75 and $4.13 \%$, respectively $(n=5)$. The detection limits $(S / N=3)$ were ca. 200 pmol.

\section{Anion-exchange HPLC of organic phosphates}

Based on examination of the hydrolysis reaction, anion-exchange HPLC of phosphoethanolamine (PEA), histidinol phosphate (His-P), Glc-1-P and $\beta$ glycerophosphate $(\beta$-Gly-P) was performed with an Asahipak NH2P-50 column using isocratic elution with $160 \mathrm{mM} \mathrm{LiCl}$ (Fig. 3). The calibration curves for compounds tested were linear in the range $30-1000 \mu \mathrm{M}$ (correlation coefficient, $0.987-0.996$ ) under the isocratic elution. When $2 \mathrm{nmol}$ each of hydrophilic organic phosphates were repeatedly injected into this system, the RSD were between 3.56 and $4.22 \%(n=5)$, respectively, the detection limits $(S / N=3)$ being $c a .250$ pmol.

The postcolumn phosphate-selective fluorescence detection system ${ }^{4}$ originally developed for hydrophobic organic phosphates was found to be applicable to hydrophilic organic phosphates by modifying the hydrolysis reagent. It is expected to have a wide application in biomedical fields.

\section{References}

1. H.-Y. Kim, T.-C. L. Wang and Y.-C. Ma, Anal. Chem., 66, 3977 (1994).

2. J. Feurle, H. Jomaa, M. Wilhelm, B. Gutsche and M. Herderich, J. Chromatogr. A, 803, 111 (1998).

3. J. X. Yan, N. H. Packer, A. A. Gooley and K. L. Williams, J. Chromatogr. A, 808, 23 (1998).

4. Y. Ikeguchi and H. Nakamura, Anal. Sci., 15, 229 (1999).

5. S. H. Kaufmann, S. Desnoyers, Y. Ottaviano, N. E. Davidson and G. G. Poirier, Cancer Res., 53, 3976 (1993).

6. M. J. Berridge, Nature [London], 365, 388 (1993).

(Received July 1, 1999) (Accepted August 19, 1999) 